\title{
Interviewer Assessments of Applicant "Fit": An Exploratory Investigation
}

\author{
Sara Rynes \\ Cornell University \\ Barry Gerhart \\ Cornell University \\ Working Paper \#89-13 \\ (Revised October 1989)
}

Center for Advanced Human Resource Studies

New York State School of Industrial and Labor Relations

Cornell University

Ithaca, N.Y. 14851-0952

(607)255-3279

Forthcoming in Personnel Psychology

This paper has not undergone formal review or approval of the faculty of the ILR School. It is intended to make the results of Center research, conferences, and projects available to others interested in human resource management in preliminary form to encourage discussion and suggestions. 


\begin{abstract}
Although both strategic management theorists and practicing recruiters endorse selecting applicants on the basis of "fit," precise delineation of fit in a selection context remains elusive. Moreover, the majority of previous work in this area has been based on anecdotes, case studies, or prescriptions rather than empirical evidence. The present investigation examines interviewers' assessments of job applicants in terms of both general and firm-specific employability (i.e., fit). Results suggest that (1) assessments of general employability differ from firm-specific assessments, (2) there is a firm-specific component to interviewers' evaluations of job applicants, and (3) interpersonal skills, goal orientation, and physical attractiveness contribute to assessments of fit (holding general employability constant), while objective qualifications (e.g., grade point average, extracurricular offices, years experience) do not. Suggestions for future research are offered.
\end{abstract}




\section{Interviewer Assessments of Applicant "Fit": An Exploratory Investigation}

The notion that it is desirable for individuals to "fit" their environments has become a basic tenet in many areas of psychology and human resource management. For example, questions concerning person-environment fit have provided much of the impetus for research in occupational psychology and vocational guidance (Holland, 1973; Spokane, 1987); recruitment (Schneider, 1976; Wanous, 1980); socialization (Fisher, 1986) and work adjustment (Lofquist \& Dawis, 1968).

Although each of the above literatures focuses to some extent on both individual and organizational outcomes, it is nevertheless fair to say that the primary emphasis has been on individual perceptions and outcomes, particularly satisfaction. To the extent that organizational outcomes are addressed in these literatures, they are typically conceptualized through the mediating effects of fit on individual attitudes, intentions, and behaviors.

Recently, however, the concept of fit has gained increasing currency on the organizational side as well, particularly in relation to employee selection. Consistent with the general trend toward contingency theories of management (e.g., Harrigan, 1983; Miles and Snow, 1978; Schuler \& Jackson, 1987), academics and consultants have increasingly recommended that job applicants be assessed in terms of their fit with the employing organization's strategies, culture, norms and values (e.g., Gerstein \& Reisman, 1983; Herbert \& Deresky, 1987; Kerr, 1982; Leontiades, 1982; Olian \& Rynes, 1984; Tichy, Fombrun \& Devanna, 1982). 
Despite the current popularity and intuitive appeal of such recommendations, the concept of fit in selection remains elusive. For example, much of the prescriptive literature is based on logical argument rather than empirical evidence (e.g., Olian \& Rynes, 1984; Snow \& Miles, 1986). Moreover, even among studies that claim an empirical foundation, the "data" are more likely to be comprised of anecdotes or case studies than conventional empirical validation results (e.g., Gerstein \& Reisman, 1983; Leontides, 1982; Ricklefs, 1979). Third, the precise dimensions of fit have not been subjected to recommended construct validation procedures (e.g., Schwab, 1980). As such, "fit" continues to elude precise, consistent definition.

The present research represents an exploratory investigation of three important, but largely unexamined, questions concerning interviewer assessments of applicant fit: (1) How (if at all) do assessments of fit differ from assessments of general employability? (2) To what extent do assessments of fit reflect something other than idiosyncratic recruiter preferences? and (3) What kinds of trait evaluations are associated with assessments of fit, holding "objective" qualifications and assessments of general employability constant? We turn now to a brief discussion of how these questions have (or perhaps more accurately, have not) been addressed in previous selection research.

\section{Applicant Fit: An Elusive Construct}

Any evaluation of the role of fit in prior selection research depends critically on how one defines it. For example, if fit is defined primarily as the correspondence between individual knowledge, skills, and abilities (KSAs) and job requirements, then the voluminous validation literature can surely be said to have addressed fit in considerable detail. 


\section{$5 \quad$ Applicant Fit}

However, most discussions of fit appear to emphasize applicant characteristics that extend far beyond the realm of job-analytically-derived KSAs. For example, Ricklefs (1979) talks about the search for candidates with appropriate "chemistry," while Schneider (1987) describes the tendency of current organizational members to hire only "right types" (e.g., individuals highly similar to themselves and current employees; see also Klimoski \& Strickland, 1977).

A cursory review of the traits commonly associated with fit, chemistry, or right types reveals such attributes as personal values, political orientation, hobbies, personality traits, attire, physical characteristics, use of leisure time, and even eating habits (e.g., Herbert \& Deresky, 1987; Mayfield \& Carlson, 1966; Ricklefs, 1979). Moreover, discussions of fit suggest that such factors become particularly important once preliminary screening establishes that all (remaining) candidates meet minimal job requirements (e.g., Ricklefs, 1979). If so, these factors may become the principal criteria for determining who, from among a pool of similarly qualified applicants, actually receives a job offer.

Although there is no universally accepted conceptualization of fit in a selection context, we believe the following statements are consistent with the bulk of (limited) previous literature on the subject: (1) although fit may encompass some elements of correspondence between individual KSAs and job requirements, the construct extends considerably beyond such immediate job-related factors; (2) assessments of fit become most important after applicants have been deemed adequately qualified to perform the work; as such, they are often the determinative assessment in decisions to extend job offers; (3) as commonly employed, the notion of fit implies a distinct firm-specific component to applicant evaluation; that is, evaluations of fit are presumed to include something more 
than just (a) assessments of "general employability" for any organization or (b) idiosyncratic reactions of individual evaluators; and (4) because many of the attributes associated with fit are interpersonally exhibited and evaluated, fit is most commonly assessed via the employment interview.

Because there is such limited empirical and construct validity evidence concerning fit in selection contexts, it is likely that future research will lead to modifications or refinements of the preceding generalizations. Nevertheless, in the interim we shall use the above framework for discussing the present investigation, as well as its relationship to previous research.

\section{Research Questions}

The first question, "How do assessments of firm-specific fit differ from assessments of general employability?" is important because there is some doubt as to whether the two constructs are in any way differentiable. For example, some authors have suggested that typical recruitment and selection procedures are really searches for a generically "ideal" candidate: someone who is not only well-qualified, but also courteous, loyal, trustworthy, and obeys the corporate laws (e.g., Schneider, 1976). Additionally, many of the anecdotal descriptions of fit (not to mention most interview evaluation forms) read like a list of "apple pie" attributes: leadership, motivation, enthusiasm, creativity, analytic abilities, warmth, intelligence, and so on. Therefore, unless these generic qualities are evaluated in a somewhat more "tailored" fashion when assessing fit (versus general employability), the concept of fit may be more a comforting fiction than a strategically-based reality.

Unfortunately, most previous interview research does not permit examination of general versus firm-specific evaluative components. This is because the vast majority of 
studies have either restricted their attention to interviewers from a single firm, or completely confounded firm and interviewer effects in multi-firm samples (e.g., Arvey \& Campion, 1982; Mayfield, 1964).

As such, it is impossible to separate interviewers' assessments into components reflecting (a) general preferences (e.g., common stereotypes applied widely across interviewers and firms; see Hakel \& Schuh, 1971); (b) firm-specific preferences (i.e., fit), and (c) idiosyncratic preferences among interviewers within a given firm (e.g., Mayfield \& Carlson, 1966). Moreover, interpretation of the dependent variable becomes clouded in such studies: where evaluation formats do not distinguish between general and firm-specific employability, it is difficult to know how much attention subjects focus on each component in formulating their overall evaluations.

The second question, "Do assessments of fit reflect anything more than the idiosyncratic preferences of individual recruiters?" is also important. For applicantorganization fit to have any of the benefits hypothesized by strategic management theorists, it must not only reflect something more than assessments of general "apple pie" attributes, but also more than individual idiosyncracies and biases. In other words, there must be a greater degree of communality among the assessments of individuals from the same firm than among individuals from different firms.

That there are wide individual differences in assessments of applicants is clear. First, previous research has shown important individual differences in both perceived importance and favorability of certain applicant attributes (e.g., Mayfield \& Carlson, 1966; Valenzi \& Andrews, 1973). Second, prior interview research has generally produced disappointing interrater reliability evidence, although estimates vary widely across studies 
(Wagner, 1949; Mayfield, 1964; Arvey \& Campion, 1982). Third, previous research suggests that most organizations spend little time training interviewers in how to evaluate applicant suitability, or what to what to tell applicants about the job or firm (e.g., Rynes \& Boudreau, 1986). In sum, given the wide variability in individual assessments and the apparent lack of organizational attention to explicitly shaping interviewers' preferences, it is possible that their evaluations reflect only general and/or idiosyncratic stereotypes, with nonexistent or insignificant firm-specific components.

Again, most previous research does not permit examination of this question. Although limited evidence suggests that interviewers from the same firm sometimes vary widely in their interpretations of specific information (e.g., Mayfield \& Carlson, 1966; Valenzi \& Andrews, 1973), the existence of certain idiosyncratic interpretations does not rule out the simultaneous coexistence of other shared perceptions due to common organizational membership. (Moreover, both of the aforementioned studies used "paper people" and generic job descriptions, neither of which would be expected to elicit much in the way of firm-specific reactions).

Indeed, recent evidence suggesting that panel interviews improve interrater reliability (Arvey \& Campion, 1982) is consistent with the notion that interviewers from a single organization agree more closely than those from different organizations. However, definitive support for this interpretation is precluded by the fact that panel interviews typically include other features believed to enhance reliability as well (e.g., structured questioning, standardized administrative procedures, and simultaneous viewing of the identical interviewee "performance"). 
The third question, "What kinds of trait evaluations are associated with fit, holding objective qualifications and general employability constant?" has also escaped empirical scrutiny. Anecdotal accounts and post-hoc speculations concerning dimensionality abound; however, methodical study of construct dimensionality is sorely lacking.

To date, few studies have been designed to permit examination of the similarities or differences between general, firm-specific, and individual-specific components of interviewer assessments. However, there are a few notable exceptions. For example, Bass (1951) studied independent ratings of applicants by managers from the same, and different, organizations (insurance and office machines). Each manager used a firm-specific structured interview to evaluate applicants for sales positions within his own firm. Ratings (of the same student) correlated .74 when made by managers from the same office machine firm, .56 for managers from the same insurance firm, but .24 and -.10 when managerial ratings were paired across companies.

Thus, Bass' results are consistent with the notion that common organizational membership enhances interviewer perceptions of applicant fit. However, there are two potentially important confounds: the use of company-specific structured interviews, and potential differences in sales positions across the two firms. The results are also based on a very small number of interviewers and only two organizations.

A second relevant study was performed by Mayfield and Carlson (1966) as part of a larger investigation of interviewer assessments. In the main study, the authors showed that certain pieces of applicant information drew highly uniform reactions from interviewers, while others generated highly diverse reactions. 
In the substudy, 31 randomly selected items describing hypothetical applicant characteristics were evaluated (in terms of favorability) by 110 insurance managers from 42 firms. Their responses were then compared with those of 77 managers from a single insurance firm. Comparisons of within-item variances revealed that for all but two items, variance for the within-firm group was as large as that for the between-firm group.

According to the authors, "The results give no indication that disagreement is greater for managers from different companies than for managers from the same company" (Mayfield \& Carlson, 1966, p. 46). It should be noted, however, that although the authors analyzed differences in item-by-item variability, they did not report any measures of average interrater agreement (e.g., reliabilities) for the between- versus within-firm groups.

Thus, results from Bass (1951) and Mayfield \& Carlson (1966) suggest conflicting conclusions regarding the presence of a firm-specific component in employability assessments. However, the studies varied in a number of respects: Bass' subjects evaluated real applicants on an overall basis in actual interviews, whereas Mayfield \& Carlson's evaluated "paper people characteristics," item by item, in an experimental setting. Moreover, both studies were based on a very small number of within-firm examinations (only one firm in Mayfield \& Carlson), and both were performed long before the recent interest in contingency theories of management and strategic notions of fit.

The present research represents an exploratory attempt to shed new light on the question of whether or not there is indeed a firm-specific component to assessments of applicant fit. Recruiters from multiple organizations provided assessments of both general employability and firm-specific employability (i.e., fit) for a two-year sample of graduating MBA job seekers. This design yielded multiple evaluations of each job candidate by 
recruiters from diverse organizations, including fifty-six instances where a given job seeker was interviewed by two or more recruiters from the same organization.

More specifically, we examine how ratings of general employability differ from assessments of fit for a particular organization, and whether interviewers from the same firm agree more in their assessments of employability (both general and firm-specific) than pairs of recruiters from different organizations. Finally, we conduct preliminary analyses to determine which subjectively evaluated applicant traits are associated with assessments of fit (and which are not), controlling for objective applicant characteristics (e.g., GPA, extracurricular offices) and assessments of general employability. Hypotheses

The first question concerns whether, or in what ways, assessments of general employability differ from assessments of firm-specific employability. Three differences were predicted a priori.

First, it was hypothesized that assessments of firm-specific employability (i.e., fit) would be lower, on average, than assessments of general employability. Previous research suggests that interviewers typically weight negative information more heavily than positive because they fear being criticized for making false positive selection errors (e.g., Springbett, 1958). Because this fear only comes into play when interviewers make judgments regarding their own firms, we expected that interviewers would apply more stringent standards in evaluating firm-specific than general employability. Additionally, one would expect that in evaluating organization-applicant fit, there would be more potentially disqualifying dimensions than when considering the more abstract question of general 
employability. Finally, it would be unlikely that interviewers would view applicants as highly suited to their firms if they did not also perceive them as generally employable.

The second hypothesis was that firm-specific evaluations would be more variable than general assessments. If organizational characteristics (culture, values, business strategies) indeed influence interviewers' judgments of fit, over and above general and idiosyncratic rater tendencies, then differences in interviewers' organizations should produce greater variance in firm-specific than general assessments.

Based on a similar logic, the third hypothesis was that inter-rater reliabilities would be lower for firm-specific than general employability assessments. Again, to the extent that differences in organizational characteristics primarily influence assessments of firm-specific fit (at least more so than general employability), this should be reflected in lower agreement (between raters from different firms) on the firm-specific employability measure.

Finally, the fact that two recruiters from the same organization sometimes (independently) interviewed the same applicant provided us with a second, and more direct, test of the notion that assessments of applicant fit contain a firm-specific component. Thus, hypothesis four predicted that recruiters from the same organization who independently interviewed the same applicant would agree more closely on firm-specific employability than when paired with recruiters from different companies who evaluated those same applicants.

Method

Sample. Data were provided by recruiters who interviewed graduating MBA students from a nationally ranked (top five to top twenty, depending on the poll) Ivy League business school during the $1987-88$ and $1988-89$ recruiting seasons. Recruiter participation 
was solicited by the Placement Director, who (truthfully) informed recruiters that their feedback would be used to (1) provide specific interviewing guidance to those students who were having difficulty generating offers, and (2) determine whether there were any areas in which the school's graduates were perceived as needing general improvement. (In general, however, applicants appear to have been highly employable. For example, in the 1987-88 season, the typical student received more than 3 job offers and accepted a job paying $\$ 47,000$ per year).

In the first recruiting season, recruiters from 89 organizations provided 1155 sets of ratings on 156 students, for an average of 7.4 evaluation sets per student. In the second season, 83 organizations provided 1104 sets of ratings on 190 students, for an average of 5.8 sets per student.

The school in question has a reputation for being heavily finance-oriented. Indeed, approximately $55 \%$ of the student sample sought positions in finance, $16 \%$ in marketing, $6 \%$ in accounting, and $23 \%$ in general management or other areas. Still, there is not much pressure for students to develop a functional specialty during the program: all students take the same core courses in the first year, and many graduate without declaring a specific major.

In most cases, interviews were arranged and allocated via applicant bidding procedures. However, at least some of the slots were set aside for "closed" interviews, where recruiters explicitly asked to interview particular students on the basis of pre-visit resume screening.

In general, it was felt (and confirmed by the Placement Director) that most students would be perceived as at least minimally qualified for the positions for which they 
interviewed (a potential "boundary condition" for assessing fit). This assumption was based on several considerations. First, students were only invited to closed interviews when recruiters had already judged that they were probably well-qualified for the position. Second, applicants would be unlikely to bid (or at least to bid many points) for interviews where they did not meet position specifications, as this would reduce the number of points available for interviews for which they were better qualified. Third, many of the positions listed involved general management or traineeships, and the school is typically regarded as producing generally trained rather than specialized business graduates.

Instruments. Following each interview, recruiters provided three kinds of assessments about each applicant: (1) ratings on ten specific trait scales (e.g. general knowledge, demonstrated leadership, warmth), (2) ratings of "overall employability," and (3) ratings of "employability in your firm (i.e., fit)." All items were measured on 5-point scales, with "5" indicating the most favorable evaluation.

Additional information about "objective" applicant characteristics (GPA, sex, number of extracurricular offices held, years of business experience, major) came from a student data base maintained by the school.

Finally, a preliminary investigation undertaken by school administrators had suggested that physical characteristics, particularly physical attractiveness, might also be associated with recruiters' evaluations. Consequently, two placement officials independently evaluated each applicant in terms of physical attractiveness and height (3-point scales: average, significantly above/below average). Officials were instructed to evaluate attractiveness in a first-impression sense (i.e., as would be judged by a recruiter meeting the individual for the first time, in interview attire). Raters were chosen for their close familiarity with nearly all 


\section{$15 \quad$ Applicant Fit}

graduating students. However, "backup" help was available, if needed, in the form of photographs on file. Interrater correlations were .50 for attractiveness, and .64 for height.

Analyses. Three types of analyses were conducted to address the question of how firm-specific employability assessments differ from general employability assessments. First, average ratings for the two evaluations were calculated and $t$-tested for significant differences. Second, their variances were computed and compared. Third, interrater reliabilities for the two measures were estimated using intraclass correlations (Ebel, 1951; Guilford, 1954).

The second question addressed the extent to which assessments of firm-specific employability reflect anything more than idiosyncratic interviewer preferences and general employability assessments. This involved comparing the interrater reliabilities of a subset of recruiters from the same firm who independently evaluated a particular applicant $(\mathrm{N}=56$ recruiter pairs) ${ }^{1}$ with "between-firm" interrater reliabilities for the same set of applicants.

More specifically, wherever same-firm interviewers rated the same candidate, withinfirm interrater correlations were calculated for both the general employability and firmspecific measures. Then, each of these interviewers was paired with another randomly selected interviewer from a different organization who also interviewed that applicant. Average values for pairs of these (across-firm) interrater correlations were then compared with the within-firm correlations. To the extent that within-firm interviewer evaluations reveal closer agreement than between-firm pairs, particularly on the firm-specific measure, support would be indicated for the presence of a firm-specific component in the applicant evaluation process. 
Finally, a number of exploratory analyses were conducted to determine what kinds of applicant characteristics predict interviewers' evaluations of firm-specific employability. Three categories of possible predictors were examined via regression analyses: objective characteristics (GPA, sex, number of extracurricular offices held, years of business experience, major); physical characteristics (average rated attractiveness, averaged rated height), and subjective interviewer assessments of the ten specific traits.

Results

Turning first to differences between assessments of general and firm-specific employability, t-tests revealed that mean assessments of firm-specific employability in the two years $(3.28,3.25)$ were lower than mean assessments of general employability (3.79, $3.79 ; \mathrm{t}=24.4$ and 24.0 , respectively). Second, using the coefficient of variation (i.e. the standard deviation/mean $\times 100$ ), firm-specific employability ratings in each of the two years $(3.20,3.63)$ were also more variable across interviewers than general employability assessments $(2.12,2.45)$. On the other hand, average interrater reliabilities for those who interviewed the same students were virtually indistinguishable for the general versus firmspecific measures (.184 vs. .179 in $1988 ; .246$ vs. .204 in 1989).

As a whole, then, our results suggest that interviewers respond differently to requests for general versus firm-specific ratings. However, based on these analyses alone, there is little support for the notion that raters from different organizations agree more closely on assessments of general employability than on firm-specific fit. Moreover, although assessments of firm-specific employability displayed greater variance than general employability, this could be explained by the fact that interviewers were less lenient on the firm-specific evaluation and, as such, used a wider portion of the scale. Thus, the question 
remains whether the greater variability in firm-specific ratings reflects anything more than idiosyncratic recruiter preferences and/or statistical artifacts.

A more appropriate method is to obtain interrater reliability estimates from recruiters within a single firm evaluating the same students. These reliabilities can then be compared to reliabilities obtained when each of these recruiters is paired with another randomly selected interviewer from a different organization who also interviewed the same applicant. This analysis revealed that within-firm pairs agreed more closely than between-firm pairs on both general and firm-specific ratings. However, the agreement was substantially more pronounced for the firm-specific (.49 vs .20$)$ than the general rating (.33 vs .24 ; with each correlation based on 56 recruiter pairs). This result, then, does suggest that interviewers from the same organization share at least some common notions about firm-specific employability or applicant fit. ${ }^{2}$

To test the extent to which objective characteristics (GPA, sex, business experience, major, and number of extracurricular offices held) explain assessments of fit, over and above assessments of general employability, firm-specific ratings were first regressed on general employability to obtain a baseline model. ${ }^{3}$ Adding objective characteristics to this model did not improve its explanatory power in either $1988\left(F_{6,573}=1.75\right)$ or $1989\left(F_{6,517}=\right.$ .82). This finding is consistent with our prior speculations that (a) candidates were, by and large, perceived as adequately qualified on an objective basis, and (b) assessments of fit involve something more than evaluation of objective qualifications.

In contrast, an analogous procedure revealed that height and attractiveness did add explanatory power to a model of fit $\left(F_{2,978}=8.73, p<.01\right)$. As shown by standardized 
regression coefficients, attractiveness $\left(\mathrm{B}_{\mathrm{att}}=.087 ; \mathrm{p}<.001\right)$ rather than height $\left(\mathrm{B}_{\text {beight }}=\right.$ .028 ; n.s.) accounted for most of the improvement in the model.

Firm-specific assessments were also regressed on the ten specific items used for student feedback (equation 1, Table 1). In addition, general employability was added to a second equation to determine whether the magnitude or statistical significance of particular coefficients would be affected (equation 2). If so, one plausible interpretation would be that general employability mediates the effects of those items on assessments of fit.

(Insert Table 1 about here)

As Table 1 indicates, several variables are statistically significant in the first equation in each of the two years. However, once general employability is controlled (equation 2), only demonstrated leadership, goal orientation, and warmth remain statistically significant in both years.

An important concern with the preceding analysis is that the collinearity among items inflates the standard errors. In addition, this collinearity suggests that a more parsimonious model could be obtained by factor analyzing the items. Thus, as an alternative strategy, we statistically removed the variance in each of the specific items accounted for by general employability, factor analyzed these residualized items, and computed factor scores. ${ }^{4}$ To determine the number of factors, a scree test was used. In addition, we looked for stability in the factor loadings matrix across the two years of data. (The descriptive statistics for these variables appear in the Appendix.) 
A four-factor solution (Table 2) using principal factor analysis and an orthogonal rotation was chosen for both years, accounting for $51 \%$ and $46 \%$ of the total variance in 1987-88 and 1988-89, respectively. ${ }^{5}$ The structure across the two years was very stable, with factor 1 apparently picking up interpersonal characteristics (interpersonal), factor 2 related to future plans and goals (goal orientation), factor 3 related to breadth of knowledge and awareness (knowledge), and factor 4 related to prior accomplishments and demonstrated leadership (accomplishments).

(Insert Tables 2 and 3 about here)

Finally, the variance in firm-specific ratings explained by general employability was statistically removed (via regression analysis) for each year's data to obtain residualized fit scores. These residuals were then regressed on the four factors. Results (Table 3) suggested that assessments of fit were most heavily associated with the interpersonal factor, followed by goal orientation and past accomplishments. In contrast, assessed knowledge appeared to have little impact on assessed fit.

\section{Discussion}

In answer to our first question, present results suggest that interviewer assessments of firm-specific employability do differ somewhat from evaluations of general employability. First, recruiters appear to be more stringent in their evaluations of firm-specific than general employability. This is not surprising, given that there are probably more dimensions on which to "disqualify" an applicant in a firm-specific context, and also that firm-specific assessments are likely to be of greater personal consequence to the interviewer (e.g., Webster, 1949; Mayfield, 1964). 
Second, firm-specific evaluations exhibit greater variability across (between-firm) interviewers than do general employability assessments. This finding is consistent with (but not probative of) the notion that differences in firm characteristics influence evaluations of firm-specific employability, over and above general evaluations of applicant characteristics and qualifications.

Further evidence of a firm-specific component to interview judgments is provided by the higher within- than across-firm interrater reliabilities for assessments of the same applicant. Moreover, although a firm-specific component appears to be present in both general and firm-specific evaluations, the effect was much stronger for the latter than the former. Thus, as hypothesized, shared organizational membership appears to influence assessments of fit to a greater extent than general employability.

Taken together, the above results suggest the importance of precisely defining in interview research which kind of "employability" interviewers are expected to assess. Previous studies have typically asked interviewers to provide a single overall rating (sometimes in conjunction with specific dimensional ratings as well), where rater and firm effects are completely confounded in the interviewer's response. As such, there is no way of knowing the extent to which subjects in previous interview research were focusing on general, as opposed to firm-specific, employability in their assessments. Moreover, previous reviews of the interview literature have not addressed the potential implications of different wordings of the overall rating scale. Thus, our results suggest that at least some of the previously reported differences in between-study findings may be attributable to different wordings of the dependent variable, and correspondingly different interpretations as to the degree of firm-specificity required in the evaluations. 
Our preliminary investigations into the determinants of firm-specific fit suggest that "objective" qualifications have little explanatory value, at least in this highly credentialed sample. This accords with our prior assumptions that (a) most of the applicants in this study were regarded as at least minimally qualified for most positions, and (b) assessments of fit (as well as general employability) involve something beyond evaluation of knowledges, skills, abilities, and past accomplishments.

Consistent with prior anecdotal reports (e.g., Ricklefs, 1979), our results suggest that a variety of subjectively assessed factors do, in fact, influence fit assessments. In particular, assessments of interpersonal skills, future goal orientation, and personal appearance all contributed significantly to these assessments, controlling for general employability. Of course, given the nature of the data, it is impossible to say precisely which aspects of, say, goal orientation had the greatest influence on recruiters, or which had the largest firmspecific (as opposed to idiosyncratic or general) component.

In summary, in contrast to most previous discussions of applicant fit, the present study provides concrete empirical evidence that assessments of fit (a) differ from assessments of general employability, and (b) reflect something more than idiosyncratic interviewer preferences. These findings have not been observed in prior interview research for two reasons: the vast majority of studies have completely confounded interviewer and firm characteristics, and have not distinguished between general and firm-specific employability.

In addition, the present research improves on the limited research that has examined firm- versus individual-specific components of applicant evaluations. For example, in comparison with Bass (1951), our results (a) are based on far more recruiters and students, (b) employ both general and firm-specific evaluations (the precise nature of Bass' overall 
rating scale is not specified), and (c) do not perfectly confound firm, job, and structured interview effects. In comparison with Mayfield and Carlson (1966), our results are based on holistic evaluations of real applicants by real recruiters in actual hiring situations, rather than on specific "paper people" attributes considered in isolation from one other. In addition, only a single firm was represented in the within-firm portion of Mayfield and Carlson's investigation.

Nevertheless, present results leave many important questions about fit unanswered. Future research in this area seems highly desirable for at least three reasons. First, practicing recruiters consistently confirm the importance of perceived "fit" in their referral and hiring decisions, particularly when applicants are at least minimally qualified (e.g., Ricklefs, 1979). Indeed, one major impetus for the present research was the frequency with which recruiters at this particular business school explained their decision processes to the Placement Director in terms of "fit." When pressed for specifics, they usually responded with lists of generic, "apple pie" attributes such as leadership, analytical abilities, motivation, warmth, enthusiasm, goal orientation, and the like. Hence, a decision was made to investigate how assessments of firm-specific fit might differ from those required by the more general measure then in use (i.e., the general employability measure in the present research).

Additionally, the strategic management literature continues to prescribe that recruiters should assess the compatibility of applicant characteristics with organizational norms, values, strategies, and traditional ways of doing business (e.g., Kerr, 1982; Miles \& Snow, 1986; Olian \& Rynes, 1984; Posner, Kouzes, \& Schmidt, 1985). Thus, both normatively and positively, the concept of firm-specific fit figures heavily in discussions of selection. 
Finally, prior research suggests that organizations devote few resources to training recruiters as to what to look for in applicants, or what to tell applicants about organizations (Rynes \& Boudreau, 1986). To the extent that recruiters and hiring managers do not receive consistent, systematic information about organizational priorities and preferences, their assessments are likely to reflect larger proportions of general and/or idiosyncratic elements, and smaller firm-specific components. In short, organizations often assume that recruiters will "absorb" the culture, rather than systematically acting to insure it.

In terms of specific recommendations, one of the top priorities for future research is to pursue additional construct definition and validation. Present results give some preliminary guidelines as to the types of factors that are more (e.g., interpersonal skills, appearance) or less important (e.g., general knowledge) in assessments of fit. However, future research is needed to determine (1) precisely which aspects of, say, interpersonal skills are most important (on average) in fit assessment, (2) whether these aspects are generally important to all interviewers, or only to some, and (3) the extent to which the perceived favorability of specific behaviors related to these aspects is consistently assessed by interviewers from various organizations (e.g., certain types of appearance might be evaluated very differently by interviewers from Vogue versus U.S. News \& World Report, yet appearance per se might be important to both).

Anecdotally, a wide variety of personal characteristics have been mentioned as possible determinants of fit: individualistic versus group orientation, attitudes toward risk, personal appearance and attire, ethnic background, extent of specialized versus general skills, and extent of previous work experience (some firms prefer it, others do not; see Ricklefs, 1979). As such, it would be helpful if future researchers were to determine 
whether these and other differences can be reliably summarized via more general dimensions. As a next step, the generalizability of such dimensions across job levels, observers (e.g., applicant versus interviewer), and time periods (pre- versus post-hire) should also be assessed.

Historically, interview researchers have discovered at least two kinds of applicant attributes: those that are evaluated rather consistently across interviewers, and those that generate highly idiosyncratic evaluations (e.g., Mayfield \& Carlson, 1966; Hakel \& Schuh, 1971). Present results (as well as the strategic management literature) suggest a third probable category: attributes that are evaluated neither completely generally nor idiosyncratically, but rather, that are evaluated more similarly by members of the same organization than different ones.

To address this question, more intensive methodologies should be employed in future investigations. For example, a reasonable first step would be to define specific aspects of organizational culture, values, and strategies that are believed to most strongly affect assessments of applicant or employee fit (see Schneider, 1975). This might be done through one-on-one interviews with multiple members of diverse organizations, or through group procedures such as or brainstorming or critical incident techniques. Similar methods could be used to generate lists of applicant attributes (e.g., political conservatism, risk taking, achievement orientation, gregariousness) that are believed to correspond to these organizational characteristics (employment security, environmental dynamism). In a similar vein, Chatman (1989), has described the use of a standardized instrument to generate applicant and organization value profiles that can be compared to assess fit. Consistent 
with our preceding discussion, however, we would expect value congruence to be only one of several key components of fit.

Once potentially relevant dimensions of organizations and applicants have been identified, experimentally-generated applicant profiles (e.g., videotapes, resumes) or face-toface role-playing applicants could be evaluated by multiple managers from companies known to have widely varying cultures, values, and business strategies (e.g., IBM versus Data General; U.S. Sprint versus AT\&T). Applicant profiles or role plays might be specifically constructed to vary along dimensions believed to be particularly important to "fitting in" with the competing cultures (e.g., group versus individualistic orientation, short versus long time perspective).

This type of design would permit examination not only as to whether incumbents of the same organizations assess fit more similarly than different ones, but also which applicant characteristics contribute most heavily to firm-specific assessments of fit. Moreover, because such a design would generate multiple within-firm evaluators per applicant (rather than only two, as in the present investigation), more powerful analysis of variance techniques could be employed to separate applicant, rater, and firm sources of variance in evaluations.

It would also be important to determine the extent to which applicants and interviewers agree on the dimensions, both organizational and individual, that are important to achieving a good fit. At present, we know little about the extent to which the dimensionality of organizational-applicant fit depends on who is making the assessment, the employer or the applicant. In addition to identifying the factors that influence fit (content), it would be useful to examine how such factors are considered in assessing fit (process). 
For example, to what extent do employers use a sequential versus compensatory model in considering general "objective" qualifications and firm-specific criteria? Are fit assessments closely linked to first impressions? Verbal protocol analysis could be a useful tool in addressing such questions.

Another area for future research would be to determine the extent to which initial assessments of fit prove to be accurate over time. Ideally, such research would be conducted longitudinally, dimensionally as well as holistically, and from the applicant's perspective as well as the employer's. For example, although it has been argued that employee dispositions or personality are stable over time (e.g., Staw \& Ross, 1985), other evidence suggests that the extent of stability varies according to changes in situational factors (Gerhart, 1987) and further, that the latter may influence personality (e.g., Kohn \& Schooler, 1982).

An additional important area for future research concerns the extent to which selecting for fit is functional, or dysfunctional, for organizations. In a sense, the question being posed here is whether or not selecting on the basis of fit is a "valid" selection procedure that actually enhances the predictability of subsequent employee (and organizational) performance.

For example, Schneider $(1983 ; 1987)$ has raised the possibility that selecting the "right types" may appear optimal in the short run (e.g., by reducing training or socialization costs), but prove dysfunctional in the longer run (via an inability to adapt to diverse or changing circumstances). A second possibility is that certain dimensions of fit are functional only in the short term, while others are functional in both the short and the 
longer term. Yet another possibility is that the long- and short-term utilities of selecting for various aspects of fit are contingency-dependent.

Unfortunately, research of this type will have to await clearer elaboration of the "fit" construct and its dimensionality (Schwab, 1980). One potentially useful distinction may be that between "supplementary" or congruent types of fit, and "complementary" fit, where "the environment is viewed as deficient in some way, and personnel decisions are made to correct the deficit by hiring an individual with complementary (incongruent) skills" (Spokane, 1987: 218). This kind of distinction is already apparent in the executive succession literature, where supplementary fit (e.g., insider succession) is thought to be desirable under most normal circumstances, but complementary fit more desirable when turnarounds are needed (e.g., Herbert \& Deresky, 1987).

Given the substantial interest among both academics and practitioners in questions of fit, it is interesting to speculate why so little empirical work has been pursued in this area. At least one explanation would seem to lie in the perceived "softness" of the construct (see also Posner, et al., 1985) relative to other selection predictors, particularly in light of postGriggs employment validation standards. Another potential explanation concerns the conventional association between assessments of fit and employment interviews, which have endured a long history of academic denigration (e.g., Webster, 1949; Mayfield, 1964; Hakel, 1982; Arvey \& Campion, 1982).

However, times are changing. For example, concerns about stagnating employee productivity and changes in labor force composition have increased employers' willingness to implement more and more varied selection devices, including ability testing, honesty testing, and personality assessments (e.g., BNA, 1986; Day \& Silverman, 1989). Second, 


\section{$28 \quad$ Applicant Fit}

recent Supreme Court decisions have shifted the burden of proof in discrimination cases squarely on the shoulders of the plaintiff, making experimentation with alternative selection techniques less risky for employers. Third, recent meta-analyses and methodological reviews of prior interview research have reached more optimistic conclusions about the potential reliability and validity of employment interviews (see Dreher, Ash \& Hancock, 1988). And fourth, valid or not, employers (including human resource and industrial psychology departments!) almost universally continue to use the interview to assess fit (Arvey \& Campion, 1982; Hakel, 1982).

If the preceding assessment is correct, then the time for serious "fit" research has finally arrived. 


\section{References}

Bass, B.M. (1951). Situational tests: Individual interviews compared with leaderless group discussions. Educational and Psychological Measurement, 11, 67-75.

Bureau of National Affairs (1986). Selection tests: Employer interest increasing. Bulletin to Management, Jan. 2, pp. 2,7.

Chatman, J.A. (1989). Improving organizational research: A model of person-organization fit. Academy of Management Review, 14, 333-349.

Day, D.V. \& Silverman, S.B. (1989). Personality and job performance: Evidence of incremental validity. Personnel Psychology, 42, 25-36.

Dreher, G.F., Ash, R.A. \& Hancock, P. (1988). The role of the traditional research design in underestimating the validity of the employment interview. Personnel Psychology, 41, 315-328.

Ebel, R. L. (1951). Estimation of the reliability of ratings. Psychometrika, 16, 407-24.

Gerhart, B. (1987). How important are dispositional factors as determinants of job satisfaction? Implications for job design and other personnel programs. Journal of Applied Psychology, 72, 366-373.

Gerstein, M. \& Reisman, H. (1983) Strategic selection: Matching executives to business conditions. Sloan Management Review, 24 33-49.

Guilford, J. P. (1954). Psychometric Methods. New York: McGraw-Hill.

Hakel, M.D. \& Schuh, A.J. (1971). Job applicant attributes judged important across seven diverse occupations. Personnel Psychology, 24, 45-52.

Hakel, M.D. (1982). Employment interviewing. In K.M. Rowland \& G.R. Ferris (eds.), Personnel Management. Boston: Allyn \& Bacon.

Harrigan, K. R. (1983) Research methodologies for contingency approaches to business 
strategy. Academy of Management Review, $\underline{8}, 398-405$.

Harvey, R. J. (1982). The future of partial correlation as a means to reduce halo in performance ratings. Journal of Applied Psychology, 67, 171-176.

Herbert, T.T. \& Deresky, H. (1987). Should general managers match their business strategies? Organizational Dynamics, 15, 3, 40-51.

Holland, J. (1973). Making vocational choices: A theory of careers. Englewood Cliffs, N.J.: Prentice-Hall.

Holzbach, R. L. (1978). Rater bias in performance ratings: Superior, self-, and peer ratings. Journal of Applied Psychology, $\underline{63}, 579-588$.

Hulin, C. L. (1982). Some reflections on general performance dimensions and halo rating error. Journal of Applied Psychology, 67, 165-170.

Kerr, J. (1982) Assigning managers on the basis of the life cycle. Journal of Business Strategy, 2, 58-65.

Klimoski, R.J. \& Strickland, W. J. (1977) Assessment centers: Valid or merely prescient? Personnel Psychology, 30, 353-361.

Kohn, M.L. \& Schooler, C. (1982). Job conditions and personality. American Journal of Sociology, $\underline{87}, 1257-1286$.

Landy, F. J., Vance, R.J., Barnes-Farrell, J. L. (1980). Statistical control of halo error in performance ratings. Journal of Applied Psychology, 65, 501-506.

Leontiades, M. (1982) Choosing the right manager to fit the strategy. Journal of Business Strategy, $\underline{3}, 58-69$.

Lofquist, L.H. \& Dawis, R.W. (1968). Adjustment to work: A psychological view of man's problems in a work-oriented society. New York: Appleton-Century-Crofts. 
Mayfield, E.C. (1964). The selection interview -- A re-evaluation of published research. Personnel Psychology, 17, 239-260.

Mayfield, E. C. \& Carlson, R. E. (1966). Selection interview decisions: First results from a long-term research project. Personnel Psychology, 19, 41-53.

Miles, R.E. \& Snow, C. C. (1978). Organizational strategy, structure and process. New York: McGraw-Hill.

Miller, E.C. (1980). Hire in haste, repent at leisure -- Team selection process at Graphic Controls. Organizational Dynamics, $\underline{8}, 2-26$.

Murphy, K. R. (1982). Difficulties in the statistical control of halo. Journal of Applied Psychology, 67, 161-164.

Olian, J.D. \& Rynes, S. L. (1984). Organizational staffing: Integrating practice with strategy. Industrial Relations, 23, 170-183.

Posner, B.Z., Kouzes, J.m. \& Schmidt, W.H. (1985). Shared values make a difference. Human Resource Management, 24, 293-309.

Ricklefs, R. (1979). The hidden hurdle: Executive recruiters say firms tend to hire "our kind of person". Wall Street Journal, Sept. 19, 1979.

Rynes, S. L. \& Boudreau, J. W. (1986). College recruiting in large organizations: Practice, evaluation, and research implications. Personnel Psychology, 39, 729-758. Schneider, B. (1975) Organizational climates: An essay. Personnel Psychology, $\underline{28}, 447-479$.

Schneider, B. (1976). Staffing Organizations. Santa Monica: Goodyear Publishing. Schneider, B. (1983). Interactional psychology and organizational behavior. In L. L. Cummings and B. M. Staw (eds.), Research in Organizational Behavior, 5, 
Greenwich, Ct.: JAI Press, 1-31.

Schneider, B. (1987). The people make the place. Personnel Psychology, 40, 437-454.

Schuler, R.S. \& Jackson, S.E. (1987). Linking competitive strategies with human resource management practices. Academy of Management Executive, 1, 207-221.

Schwab, D. P. (1980). Construct Validity in organizational behavior. In L.L.

Cummings \& B.M. Staw (ds.), Research in Organizational Behavior, 2 , Greenwich, CT.: JAI Press, 3-43.

Snow, C.C. and Miles, R. E. (1986). Organizational strategy, design, and human resources management. In S. L. Rynes \& G. T. Milkovich (eds.), Current Issues in Human Resource Management, Plano, TX.: Business Publications, Inc., 60-69. Springbett, B.M. (1958). Factors affecting the final decision in the employment interview. Canadian Journal of Psychology, 12, 13-22.

Staw, B.M. (1986) Organizational psychology and the pursuit of the happy/productive worker. California Management Review, 28, 40-53.

Staw, B.M. \& Ross, J. (1985). Stability in the midst of change: A dispositional approach to job attitudes. Journal of Applied Psychology, 70, 469-480.

Tichy, N. M., Fombrun, C. \& Devanna, M. A. (1982) Strategic human resource management, Sloan Management Review, 23, 47-61.

Valenzi, E. \& Andrews, I.R. (1973) Individual differences in the decision processes of employment interviewers. Journal of Applied Psychology, 58, 49-53.

Wagner, R. (1949). The employment interview: A critical summary. Personnel Psychology, 2, 17-46.

Wanous, J. P. (1980). Organizational entry: Recruitment, selection, and socialization of Newcomers. Reading, MA.: Addison-Wesley. 


\section{Authors' Notes}

Authors contributed equally to this project. We wish to thank Laurie Foltman and James Schmotter of the Johnson Graduate School of Business, Cornell University for their contribution to the study. We also wish to thank the School of Industrial and Labor Relations, the Center for Advanced Human Resource Studies, and the U.S. Army Research Institute (Contract SRFC-MDA903-87-K0001) for financial and staff support for this project. However, the authors retain sole responsibility for the views and findings contained herein. 
Applicant Fit

\section{Footnotes}

1. In each instance where records indicated that two same-firm recruiters had interviewed the same student, the firm was called to verify whether or not the interviews and ratings were truly conducted independently (as opposed, for example, to panel interviews). In the vast majority of cases, they were; where they were not, they were eliminated from further analysis. Of the remaining firms, in all but one case, the two recruiters from the same firm were sent to interview applicants for similar jobs, but for different locations and on different days. Given that fit assessments are likely to include both job-specific and firmspecific components, our observed interrater correlations are likely to underestimate the agreement that would be obtained where multiple recruiters from the same firm interviewed applicants for the exact same job. In fact, although the sample size is too small to be conclusive, the one firm (a financial services firm) where multiple recruiters did interview for the exact same job seemed to show greater interrater agreement, particularly on the firm-specific employability measure. Follow-up conversations with the recruiting coordinator (a line manager) made clear that she believed such agreement was a result of the following factors: their recruiters (also line managers) received interview training, were provided with a common list of attributes to look for in applicants, and were hiring for a special general finance rotation program involving four six-month assignments, which they themselves had already completed.

2. The sample of applicants interviewed by two recruiters per firm did not differ from the broader sample in terms of either firm-specific ratings (mean $=3.3, \mathrm{SD}=1.1$ versus mean $=3.3, \mathrm{SD}=1.0$ ) or general employability ratings (mean $=3.8, \mathrm{SD}=.8$ versus mean $=3.8$, $\mathrm{SD}=.8$ ). 
3. The sample sizes for these analyses were reduced because some measures (e.g. offices held) depended on student responses to a post-graduation questionnaire with a response rate of approximately $60 \%$.

4. See Holzbach (1978), Landy, Vance, Barnes-Farrell (1980), Murphy (1982), Hulin (1982), and Harvey (1982) for discussions of this procedure.

5. Principal components analysis showed that the corresponding four components had eigenvalues of at least 1.0 in each year. (Eigenvalues from principal components analysis do not correspond to those from principal factor analysis). 
Table 1

Regression of Firm-specific Fit on Item Ratings and General Employability

\begin{tabular}{|c|c|c|c|c|}
\hline & \multicolumn{2}{|c|}{1988} & \multicolumn{2}{|c|}{1989} \\
\hline & $\underline{\mathrm{Eq} .1}$ & $\underline{\mathrm{Eq}} .2$ & Eq.1 & Eq.2 \\
\hline General Employability & N/A & $.341 * * *$ & N/A & $.566 * * *$ \\
\hline Awareness & $.082 *$ & .031 & .056 & .028 \\
\hline Knowledge & .009 & .002 & .015 & -.048 \\
\hline Accomplishments & $.008 * *$ & .018 & .041 & -.021 \\
\hline Leadership & $.084 * *$ & $.055^{*}$ & $.121 * * *$ & $.076^{*}$ \\
\hline Goals & $.136 * * *$ & $.096 *$ & $.096^{*}$ & $.097^{*}$ \\
\hline Plans & $.189 * * *$ & $.156^{* * *}$ & $.099 *$ & .026 \\
\hline Appearance & .043 & .006 & .034 & -.001 \\
\hline Warmth & $.175^{* * *}$ & $.148 * * *$ & $.093 * *$ & $.079 * *$ \\
\hline Verbal Skills & $.093 * *$ & .047 & $.233^{* * *}$ & $.083^{* *}$ \\
\hline Listening Skills & $.106 * * *$ & $.092 * * *$ & .042 & -.015 \\
\hline $\mathbf{R}^{2}$ & $.614^{* * *}$ & $.657 * * *$ & $.426^{* * *}$ & $.575^{* * *}$ \\
\hline
\end{tabular}

Note: Entries are standardized regression coefficients.

$* \mathrm{p}<.05 \quad * * \mathrm{p}<.01 \quad * * * \mathrm{p}<.001$ 


\section{Table 2}

Results from Principal Factor Analysis of Interview Rating Dimensions

\begin{tabular}{|c|c|c|c|c|}
\hline & \multicolumn{4}{|c|}{ Factor Loadings 1988 (1989) } \\
\hline & Factor 1 & Factor 2 & Factor 3 & Factor 4 \\
\hline Listening & $66(61)$ & $09(09)$ & $16(07)$ & $07(10)$ \\
\hline Warmth & $63(62)$ & $03(01)$ & $01(00)$ & $07(06)$ \\
\hline Verbal & $59(59)$ & $17(11)$ & $23(12)$ & $06(03)$ \\
\hline Appearance & $46(50)$ & $12(14)$ & $10(04)$ & $09(00)$ \\
\hline Goals & $14(19)$ & 77(72) & $21(14)$ & $21(20)$ \\
\hline Plans & $19(15)$ & 76(73) & $19(19)$ & $22(19)$ \\
\hline Knowledge & $19(07)$ & $19(15)$ & $73(68)$ & $23(22)$ \\
\hline Awareness & $19(11)$ & $19(15)$ & $71(68)$ & $14(16)$ \\
\hline Accomp. & $13(04)$ & $14(12)$ & $19(21)$ & $61(62)$ \\
\hline Leadership & $13(06)$ & $20(20)$ & $10(15)$ & $60(61)$ \\
\hline Eigenvalue & $3.2(2.5)$ & $.89(.54)$ & $.59(.60)$ & $.46(.62)$ \\
\hline
\end{tabular}

Note: Decimals for factor loadings omitted.

$* \mathrm{p}<.05 \quad * * \mathrm{p}<.01 \quad * * * \mathrm{p}<.001$ 


\section{$38 \quad$ Applicant Fit}

Table 3

Regression of Residualized Fit Score on Factor Scores

\begin{tabular}{lll}
\hline & $\underline{1988}$ & $\underline{1989}$ \\
Interpersonal & $.320^{* * *}$ & $.258^{* * *}$ \\
Goal Orientation & $.257^{* * *}$ & $.215^{* * *}$ \\
Knowledge & $.065^{*}$ & .042 \\
Accomplishments & $.133^{* * *}$ & $.157^{* * *}$ \\
$\mathrm{R}^{2}$ & .237 & .174 \\
\hline
\end{tabular}

Note: Entries are standardized regression coefficients.

$* \mathrm{p}<.05 \quad * * \mathrm{p}<.01 \quad * * * \mathrm{p}<.001$ 
Appendix

Means, Standard Deviations, and Intercorrelations

\begin{tabular}{|c|c|c|c|c|c|c|c|c|c|c|c|c|c|c|c|c|c|}
\hline & & \multicolumn{2}{|c|}{1988} & \multicolumn{12}{|c|}{ Intercorrelations } & \multicolumn{2}{|c|}{1989} \\
\hline & & Mean & SD & (1) & (2) & (3) & (4) & (5) & (6) & (7) & (8) & (9) & $(10)$ & $(11)$ & $(12)$ & Mean & SD \\
\hline AWARE & (1) & 3.79 & .72 & 1.00 & .829 & .572 & .514 & .634 & .628 & .490 & .463 & .605 & .524 & .645 & .597 & 3.82 & .74 \\
\hline know & (2) & 3.74 & .77 & .808 & 1.00 & .623 & .540 & .631 & .633 & .491 & .440 & .599 & .548 & .632 & .589 & 3.75 & .76 \\
\hline ACCOMP & (3) & 3.83 & .82 & .598 & .628 & 1.00 & .710 & .592 & .604 & .445 & .482 & .513 & .472 & .642 & .587 & 3.80 & .85 \\
\hline LEADER & (4) & 3.56 & .92 & .587 & .592 & .743 & 1.00 & .592 & .596 & .437 & .458 & .486 & .439 & .597 & .571 & 3.56 & .88 \\
\hline GOAL & (5) & 3.62 & .89 & .608 & .617 & .593 & .625 & 1.00 & .874 & .482 & .450 & .585 & .505 & .656 & .663 & 3.59 & .87 \\
\hline PIAAN & (6) & 3.60 & .91 & .639 & .627 & .603 & .623 & .851 & 1.00 & .521 & .475 & .593 & .523 & .664 & .679 & 3.60 & .89 \\
\hline APPEAR & (7) & 3.83 & .75 & .489 & .452 & .417 & .424 & .524 & .539 & 1.00 & .565 & .557 & .519 & .560 & .529 & 3.95 & .73 \\
\hline WARMTH & (8) & 3.88 & .83 & .442 & .423 & .410 & .425 & .479 & .462 & .592 & 1.00 & .593 & .647 & .551 & .592 & 3.94 & .82 \\
\hline VERBAI & (9) & 3.81 & .90 & .583 & .564 & .505 & .520 & .598 & .596 & .619 & .616 & 1.00 & .689 & .635 & .621 & 3.82 & .92 \\
\hline IISTEN & $(10)$ & 3.79 & .86 & .536 & .516 & .517 & .494 & .581 & .559 & .560 & .652 & .704 & 1.00 & .565 & .595 & 3.85 & .78 \\
\hline GEN EMPP & (11) & 3.80 & .81 & .696 & .680 & .663 & .655 & .695 & .706 & .604 & .567 & .712 & .655 & 1.00 & .739 & 3.85 & .78 \\
\hline FIRM EMP & (12) & 3.301 & 1.05 & .611 & .594 & .597 & .621 & .684 & .675 & .537 & .559 & .668 & .624 & .775 & 1.00 & 3.37 & 1.02 \\
\hline
\end{tabular}

1988 correlations are above the diagonal (N = 1145); 1989 correlations are below the diagonal (N = 1087) 